\title{
Comments on the article "Effectiveness and safety of tofacitinib in rheumatoid arthritis: a cohort study"
}

\author{
Thuy Nhu Thai ${ }^{*}$ (D) and Ghadeer K. Dawwas
}

We read with interest the study published by de Ávila Machado et al. regarding the comparative effectiveness and safety of tofacitinib and non-tumor necrosis factor (TNF) inhibitors among patients with rheumatoid arthritis (RA) [1]. Although the study findings are very interesting, there are several concerns in regard to the study design. First, the study used an exposure definition that caused RA disease severity to be heterogeneous between and within the comparison groups. According to RA treatment guidelines, patients at an early stage of RA are recommended to start a combination of DMARDs, or to initiate TNF inhibitors, or non-TNF inhibitors after the failure of DMARDs monotherapy. On the other hand, patients with an established RA are recommended to use a combination of DMARDs, DMARDs with TNF inhibitors, DMARDs with non-TNF inhibitors, or DMARDs with tofacitinib [2]. Therefore, the exposure definition used in this study may reflect RA patients at different stages of disease severity which has the potential of introducing bias in the observed estimates.

For example, to initiate tofacitinib, patients have to fail the other treatment approaches causing them to be at an advanced RA stage when compared with those in the DMARDs group who are more likely to have used methotrexate only before. Additionally, it was not clear why the authors grouped together patients who combined TNF inhibitors with DMARDs along with patients who used TNF inhibitors alone. This exposure group might be comprised of two different patients' populations since it is often recommended that patients combine TNF inhibitors and DMARDs after treatment failure with TNF inhibitors alone [2].

Second, the medication possession ratio (MPR) is known to be a valid measure of adherence of a single medication. However, MPR tends to overestimate adherence when patients use more than one medication [3]. Accordingly, the observed adherence of patients who used two or more DMARDs are more likely to be overestimated. The proportion of days covered is suggested as a better alternative measure of adherence in this situation [3]. Third, the models evaluating the effectiveness and safety omitted the adjustment of important variables. For example, the authors did not adjust for current methotrexate use which may impact the observed treatment effectiveness or factors that may increase the risk of infections such as human immunodeficiency virus(HIV) or the use of immunosuppressant drugs.

\section{Authors' response}

\section{Marina Amaral de Ávila Machado, Cristiano Soares de Moura, Steve Ferreira Guerra, Michal Abrahamowicz and Sasha Bernatsky}

We thank Thai and Dawwas for their interest in our work on the effectiveness and safety of tofacitinib in RA [1] and we are pleased to clarify some aspects of our study.

The first comment concerned comparability of exposure groups. We selected patients previously treated with methotrexate and other newly dispensed DMARDs,

\footnotetext{
* Correspondence: thuythai@ufl.edu

Department of Pharmaceutical Outcomes and Policy, College of Pharmacy, University of Florida, Gainesville, Florida, USA
}

biologics, and tofacitinib between 2011 and 2014. We applied a new-user design, such that all patients in our study were initiating a second RA therapy after failure or intolerance of methotrexate. We defined four comparison groups: DMARDs, TNF inhibitors with or without DMARDs, non-TNF biologics with or without DMARDs, and tofacitinib with or without DMARDs. Non-TNF biologics comprised the reference group, given our particular interest in assessing subsequent therapies in RA. We 
allowed the groups to be concomitantly exposed to DMARDs since, in the real world, it is not uncommon for these drugs to be given in combination, for example with anti-malarial drugs or other DMARDs. Previous studies have applied similar approach $[4,5]$. Upon further review of our paper, we noticed an inconsistency in the fourth sentence of the second paragraph of the Methods section that may have contributed to a misunderstanding. We stated that "We selected individuals with no use of these medications any time before cohort entry (minimum 12 months), although previous use of DMARDs was allowed for individuals in the biologic and tofacitinib groups." In fact, patients in the biologic and tofacitinib groups who had been prescribed DMARDs before cohort entry were considered in the DMARD group.

Regarding the second point about the adherence of patients on combination therapy with DMARDs, we clarify that the medication possession ratio (MPR) was measured separately for the index DMARD (identified at the pharmacy claim at cohort entry) and for methotrexate. Then, a given patient was considered highly adherent for the combined therapy if the MPR was $\geq 80 \%$ for the DMARD or for methotrexate.

Finally, the authors questioned the omission of some potential confounders in the models. We did not adjust the effectiveness model for current methotrexate use because the addition of methotrexate between 4 and 12 months of follow-up was part of the outcome definition (criterion 3 of the algorithm). Regarding infection risk, the safety models were adjusted for previous and current use of glucocorticoids, and previous hospitalized infections [6]. We also adjusted for Charlson comorbidity index, which includes AIDS/HIV.

\section{Authors' contributions}

TNT and GD discussed the study concept, wrote the letter, and reviewed the final version. Both authors read and approved the final manuscript.

Ethics approval and consent to participate

Not applicable.

Consent for publication

Not applicable.

\section{Competing interests}

The authors declare that they have no competing interests.

\section{Publisher's Note}

Springer Nature remains neutral with regard to jurisdictional claims in published maps and institutional affiliations.

Published online: 29 August 2018

\section{References}

1. Machado MAÁ, Moura CS, Guerra SF, Curtis JR, Abrahamowicz M, Bernatsky S. Effectiveness and safety of tofacitinib in rheumatoid arthritis: a cohort study. Arthritis Res Ther. 2018;20(1):60

2. Singh JA, Saag KG, Bridges SL Jr, et al. 2015 American College of Rheumatology Guidelines for the treatment of rheumatoid arthritis. Arthritis Rheumatol (Hoboken, NJ). 2016;68(1):1-26.
3. Martin BC, Wiley-Exley EK, Richards S, Domino ME, Carey TS, Sleath BL. Contrasting measures of adherence with simple drug use, medication switching, and therapeutic duplication. Ann Pharmacother. 2009;43(1):36-44.

4. Solomon DH, Shadick NA, Weinblatt ME, Zak A, Frits M, Franklin JM. Drug safety analyses in a rheumatoid arthritis registry: application of different approaches regarding timing of exposure and confounder measurement. Arthritis Res Ther. 2017;19(1):130.

5. Gomez-Reino JJ, Carmona L, BIOBADASER Group. Switching TNF antagonists in patients with chronic arthritis: an observational study of 488 patients over a four-year period. Arthritis Res Ther. 2006;8(1):R29.

6. Widdifield J, Bernatsky S, Paterson JM, Gunraj N, Thorne JC, Pope J, et al. Serious infections in a population-based cohort of 86,039 seniors with rheumatoid arthritis. Arthritis Care Res (Hoboken). 2013;65(3):353-61. 